\title{
Effect of Oxidative Stress in Semen, Follicular Fluid, and Embryo Culture Medium on the Outcome of Assisted Reproduction
}

\author{
Nooman Sallam, ${ }^{1}$ MSc, Mostafa Hegab, ${ }^{1}$ PhD, Fahd El-Omda, ${ }^{1}$ PhD, Dalal El-Kaffash, ${ }^{2}$ PhD.
}

\section{* Corresponding Author: \\ Nooman Sallam \\ nooman sallam@hotmail.com}

Received for publication June 14 , 2021; Accepted July 24, 2021; Published online July 24, 2021, 2021.

Copyright The Authors
published by Al-Azhar
University, Faculty of Medicine,
Cairo, Egypt. Users have the
right to read, download, copy,
distribute, print, search, or link
to the full texts of articles under
the following conditions:
Creative Commons Attribution-
Share Alike 4.0 International
Public License (CC BY-SA 4.0).
doi: 10.21608/aimj.2021.79536.1495.
1. Obstetrics and Gynecology
Department, Faculty of Medicine,
Al-Azhar University, Cairo, Egypt.
2. Clinical Pathology Department,
Faculty of Medicine, Alexandria
University, Alexandria, Egypt.

Copyright The Authors University, Faculty of Medicine, Cairo, Egypt. Users have the right to read download copy, Share Alike 4.0 International doi: $10.21608 /$ aimj.2021.79536.1495.

${ }^{1 .}$ Obstetrics and Gynecology Department, Faculty of Medicine, Anar University, Cairo, Egyp

Faculty of Medicine, Alexa
University, Alexandria, Egypt.

\begin{abstract}
Background: Although many advances have been made in the field of assisted reproduction, the clinical outcomes remain less than desirable. Oxidative stress has been recently blamed on both male and female partners.

Aim of the study: This work aimed to study the effect of oxidative stress in semen, follicular fluid, and embryo culture medium on the outcome of assisted reproduction.

Patients and Methods: Fifty couples with unexplained infertility were subjected to a combined treatment of IVF and ICSI. Oxidative stress was evaluated by measuring the oxidative-reduction potential (ORP) in the native semen sample, the follicular fluid pool and the spent culture medium of individually IVF cultured embryos.

Results: ORP in the native semen was found to be a good predictor of fertilization and clinical pregnancy in IVF with a cut-off point of 1.57 and $0.75 \mathrm{mV} / 10^{6}$ sperm $/ \mathrm{ml}$, respectively. There was also a positive and highly significant correlation between the ORP levels in the follicular fluid pool and the fertilization rate $(r=0.4217 ; P=0.002288)$, but there was no statistically significant difference between ORP in the follicular fluid pool of women who became pregnant and those who did not $(P=$ $0.997425)$. In addition, the mean ORP in the spent culture medium of women who became pregnant $(215.78 \mathrm{mV} / \mathrm{ml} \pm 38.52)$ was lower than in those who did not become pregnant $(229.22 \mathrm{mV} / \mathrm{ml} \pm 31.56)$ but the difference was also not statistically significant $(\mathrm{P}=0.3170)$.

Conclusion: It is concluded that ORP in the native semen and in the spent culture medium in IVF are good predictors of fertilization and clinical pregnancy and that ORP in the follicular fluid is not.
\end{abstract}

Keywords: Assisted reproduction; ICSI; IVF; oxidation-reduction potential; oxidative stress; unexplained infertility

Disclosure: The authors have no financial interest to declare in relation to the content of this article. The Article Processing Charge was paid for by the authors.

Authorship: All authors have a substantial contribution to the article.

\section{INTRODUCTION}

Despite various developments in the field of assisted reproduction, the results of IVF and ICSI are still unsatisfactory. For example, in the 421,659 ART cycles performed in Europe in 2014, the clinical pregnancy rate and the live birth rate per retrieval were $29.3 \%$ and $22.3 \%$, respectively ${ }^{1}$. Similarly, the number of ART cycles performed in the USA in 2016 was 86,237 with a clinical pregnancy rate and a live birth rate of $30.9 \%$ and $25.1 \%$, respectively ${ }^{2}$. Various factors have been blamed for these less than satisfactory results including oxidative stress.

Oxidative stress results from an imbalance between the production of reactive oxygen species and the antioxidant capacity of the body. Reactive oxygen species (ROS) are oxygen free radicals and are normal products of cellular metabolism. They are produced as byproducts in the mitochondria during the enzymatic reduction of oxygen to produce energy ${ }^{3}$. In small amounts, ROS play a favorable role in hemostasis and the proper function of the cardiovascular and immune system. In human reproduction, they play a physiological role in the process of sperm capacitation, acrosome reaction, sperm hyperactivation and sperm oocyte binding ${ }^{4}$. However, in excessive amounts, ROS can be detrimental to many body functions.

To counteract the possibility of cellular damage by excessive production of ROS, antioxidants pathways exist in the human body. These include enzymatic and non-enzymatic pathways. They act by scavenging the excess ROS and allowing a balance to be achieved between beneficial and detrimental effects of ROS. Under normal circumstances, a 
balance is kept between the amount of ROS generated and the antioxidant capacity of the cells. Oxidative stress results when the generation of ROS supersedes the capacity of the antioxidant pathways to prevent or repair the harmful effects of the excess radicals. In general, harmful effects of ROS on the cells include (1) damage of DNA or RNA (DNA/RNA fragmentation), (2) oxidation of polyunsaturated fatty acids in lipids including cell membranes (lipid peroxidation), (3) oxidation of amino acids in proteins (oxidative degradation) and (4) deactivation of specific enzymes by oxidation of co-factors (oxidative deactivation).

Consequently, the aim of this work was to explore the role of oxidative stress in assisted reproduction in 3 particular areas (1) the role of oxidative stress in male infertility, (2) the role of oxidative stress in female infertility, and (3) oxidative stress as a method of embryo selection.

\section{PATIENTS AND METHODS}

The study was conducted between October 2017 and July 2020. A total of 216 couples were recruited for the study. All couples suffered from unexplained infertility for at least 2 years. Unexplained infertility was diagnosed on the basis of (1) a normal semen fluid analysis according to WHO criteria (WHO, $5^{\text {th }}$ edition, 2010), (2) evidence of ovulation by midluteal plasma progesterone concentration of $=>10$ $\mathrm{ng} / \mathrm{ml}$ and (3) patent tubes by hysterosalpingography or laparosocopy. Of the 216 recruited couples, 166 were excluded for the following reasons: poor response to stimulation (12 couples), <2 oocytes retrieved (68 couples), abnormal semen analysis was abnormal on the day of the operation (52 couples), lost to follow-up (15 couples) while 19 couples refused to participate. The study was therefore conducted on the remaining 50 couples.
Of the 50 couples enrolled in the study, fertilization by classical IVF was performed for 216 MII oocytes and was successful in 28 cases resulting in 72 embryos (fertilization rate $=33.3 \%$ ) and 6 clinical pregnancies (clinical pregnancy rate $=21.4 \%$ ). In the other 22 patients no fertilization occurred by classical IVF and embryos resulting from ICSI were replaced resulting in 14 clinical pregnancies (clinical pregnancy rate $=63.6 \%$ )

Oxidative stress was evaluated by measuring the oxidative-reduction potential (ORP) using the MiOXSYS platform (Aytu Biosciences, Englewood, CO, USA) in the following biological fluids: (1) the native semen sample, (2) the follicular fluid pool, and (3) the spent culture medium of individually IVF cultured embryos. The MiOXSYS System is based on electrochemical technology. The biological fluid sample is placed on the sensor which is theninserted into the analyzer. The resulting static ORP (sORP) measurement displayed reflects the balance between the reactive oxygen species and the antioxidant capacity in the analyzed specimen and is expressed in millivolts/second. The sample analysis is completed in approximately 2 minutes.

All statistical tests were performed using MedCalc Statistical Software version 19.1 (MedCalc Software bv, Ostend, Belgium; https://www.medcalc.org; 2019). Data were tested for normality using the Kolmogorov-Smirnov test before applying the appropriate statistical tests. A Pvalue of $<0.05$ was considered to be statistically significant.

\section{RESULTS}

\section{Demographic data}

The demographic and basic data of the studied couples are shown in (Table 1).

\begin{tabular}{|l|l|l|}
\hline & Mean $( \pm$ SD) & Range \\
\hline Female partner age (years) & $29.0( \pm 5.0)$ & $21-38$ \\
\hline Male partner age (years) & $34.3( \pm 5.5)$ & $28-50$ \\
\hline Duration of infertility (years) & $4.96( \pm 2.2)$ & $3-10$ \\
\hline Mid-luteal plasma progesterone $(\mathrm{ng} / \mathrm{ml})$ & $14.8( \pm 5.5)$ & $10.2-26.8$ \\
\hline Sperm count $(\mathrm{million} / \mathrm{ml})$ & $68.7( \pm 28.8)$ & $20-113$ \\
\hline Sperm progressive motility $(\%)$ & $48.8( \pm 20.9)$ & $32-85$ \\
\hline Strict sperm morphology $(\%)$ & $8.7( \pm 5.0)$ & $4-20$ \\
\hline Oocyte score & $1.86( \pm 0.47)$ & $0.96-2.71$ \\
\hline ORP in the native semen $\left(\mathrm{mV} / 10^{6} \mathrm{sperm} / \mathrm{ml}\right)$ & $1.07( \pm 1.29)$ & $0.17-4.75$ \\
\hline ORP in the processed semen $\left(\mathrm{mV} / 10^{6} \mathrm{sperm} / \mathrm{ml}\right)$ & $3.05( \pm 1.86)$ & $0.39-6.94$ \\
\hline ORP in the follicular fluid $(\mathrm{mV} / \mathrm{ml})$ & $107.74( \pm 38.18)$ & $38.21-168.9$ \\
\hline ORP in serum $(\mathrm{mV} / \mathrm{ml})$ & $97.99( \pm 18.02)$ & $77.5-159.8$ \\
\hline ORP in the native culture medium $(\mathrm{mV} / \mathrm{ml})$ & $216.72 \pm 7.89$ & $207.0-225.5$ \\
\hline
\end{tabular}

Table 1: Demographic

criteria

of the

studied

patients.

\section{ORP in the native semen}

The mean $( \pm \mathrm{SD})$ fertilization rate in the IVF studied patients was $35.5 \%( \pm 36.6)$. There was a weak negative correlation between the ORP in the native semen and the fertilization rate in the group of oocytes submitted to IVF $(r=-0.0524 ; P=0.719866)$. This is shown in (Figure 1). 
However, when the fertilization rate was stratified into $=>50 \%$ and $<50 \%$ fertilization rate, the mean $( \pm$ SD) ORP in the native semen in couples with $=>50 \%$ IVF fertilization was $1.02 \pm 0.1 \mathrm{mV} / 10^{6} \mathrm{sperm} / \mathrm{mL}$ which is significantly lower than in couples with $<50 \%$ fertilization $\left(2.05 \pm 0.7 \mathrm{mV} / 10^{6} \mathrm{sperm} / \mathrm{mL}\right)$ $(\mathrm{P}<0.02)$.

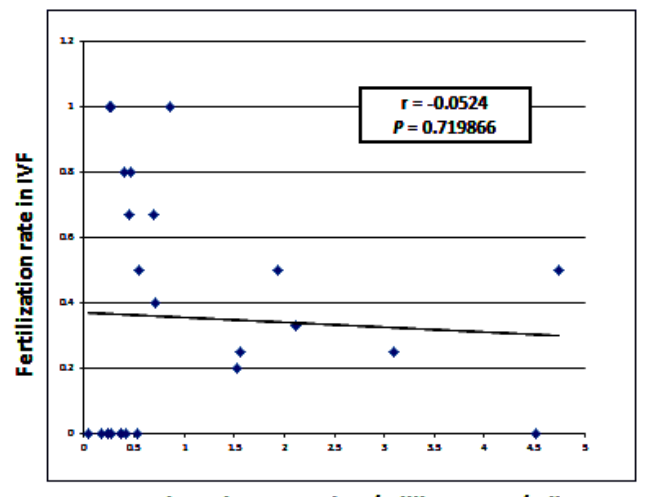

ORP in native semen (mV/million sperm/ml)

Fig 1: Correlation between the ORP in the native semen and the fertilization rate in IVF.

The ORP in the native semen was then evaluated as a predictor of fertilization in IVF. A receiver operating characteristic curve (ROC) was constructed and the area under the curve was found to be equal to 0.854167 and the cut-off point at 1.57 $\mathrm{mV} / 10^{6}$ sperm/ml (figure 2).

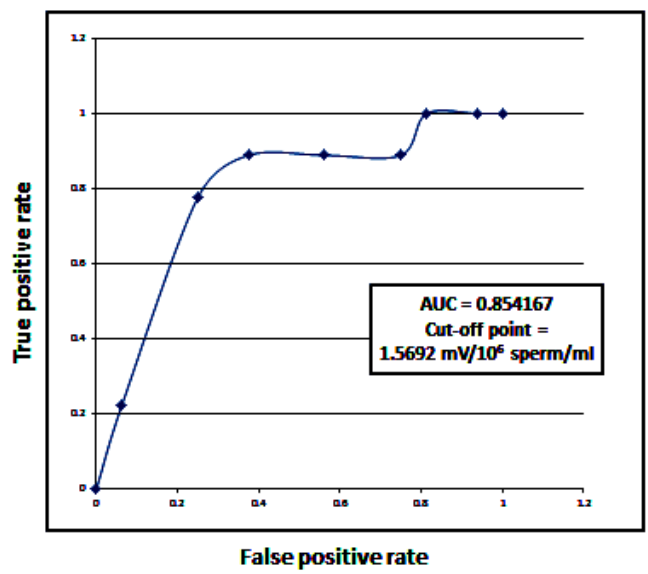

Fig 2: ROC curve for ORP in the native semen as a predictor of fertilization.

There was also a highly significant difference in the ORP of the native semen between couples that achieved a pregnancy $(0.3334 \pm 0.1719$ $\mathrm{mV} / 10^{6} \mathrm{sperm} / \mathrm{ml}$ ) and those who did not achieve a pregnancy $\left(1.5723 \pm 1.48 \mathrm{mV} / 10^{6} \mathrm{sperm} / \mathrm{ml}\right)$ in the IVF model $(\mathrm{P}=0.006102)$ The ORP in the native semen was also evaluated as a predictor of pregnancy in IVF. The ROC curve showed that the area under the curve was found to be equal to 0.8 and the cut-off point at $0.75 \mathrm{mV} / 10^{6}$ sperm $/ \mathrm{ml}$ (Figure 3 ).

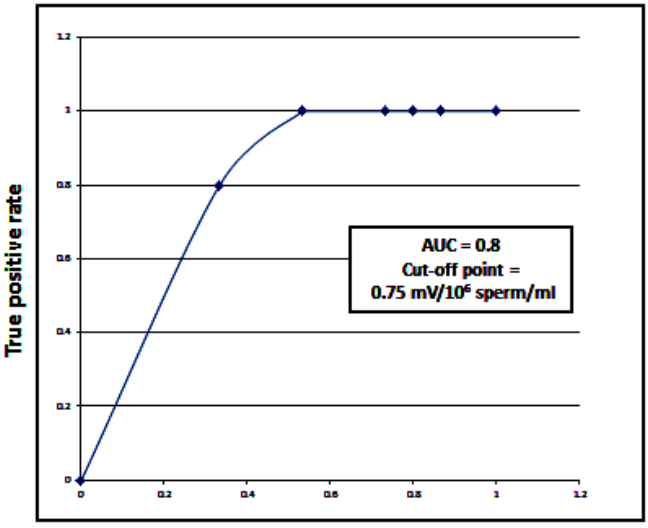

False positive rate

Fig 3: ROC curve for ORP in the native semen of pregnant and non-pregnant patients.

\section{ORP in the follicular fluid}

The mean $( \pm \mathrm{SD})$ ORP in the follicular fluid pools of the studied patients was higher [122.6111 ( \pm $41.3657)]$ in women who achieved $50 \%$ fertilization or more compared to those who did not $[98.81333$ ( \pm 7.141778)]. However, this difference is not statistically significant $(P=0.163254)$. There was a weak positive but significant correlation between the ORP in the follicular fluid and the fertilization rate in the group of oocytes submitted to IVF ( $r=0.4217$; $P=0.002288$ ). This is shown in (Figure 4).

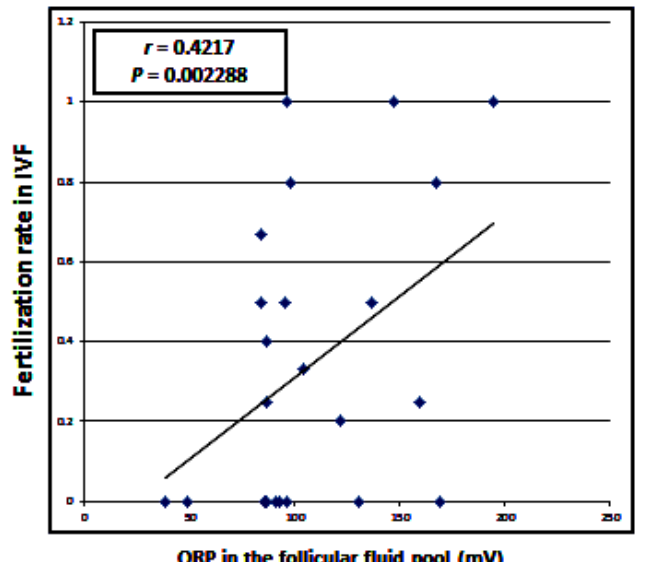

Fig 4: Correlation between the ORP in the follicular fluid pool and the fertilization rate.

However, there was no statistically significant difference between the mean $( \pm \mathrm{SD})$ ORP of the follicular fluid pool of women who became pregnant $[107.77( \pm 45.47) \mathrm{mV} / \mathrm{ml}]$ and those who did not become pregnant $[107.71( \pm 26.72) \mathrm{mV} / \mathrm{ml}](P=$ $0.997425)$. The ORP in the follicular fluid was also evaluated as a predictor of $50 \%$ fertilization of the retrieved oocytes in IVF. Receiver operating characteristic curve analysis was conducted and the area under the curve was found to be equal to 0.6593 and the cut-off point at $110 \mathrm{mV} / \mathrm{ml}$ as shown in (Figure 5). 


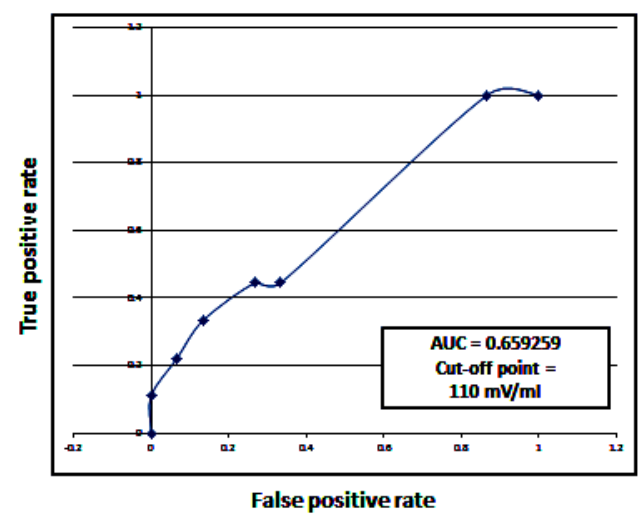

Fig 5: ROC curve for ORP in the follicular fluid as a predictor of fertilization.

The ORP in the follicular fluid pool was also evaluated as a predictor of pregnancy in IVF. Receiver operating characteristic curve analysis was conducted and the area under the curve was found to be equal to 0.6557 and the cut-off point at $90 \mathrm{mV} / \mathrm{ml}$ (Figure 6).

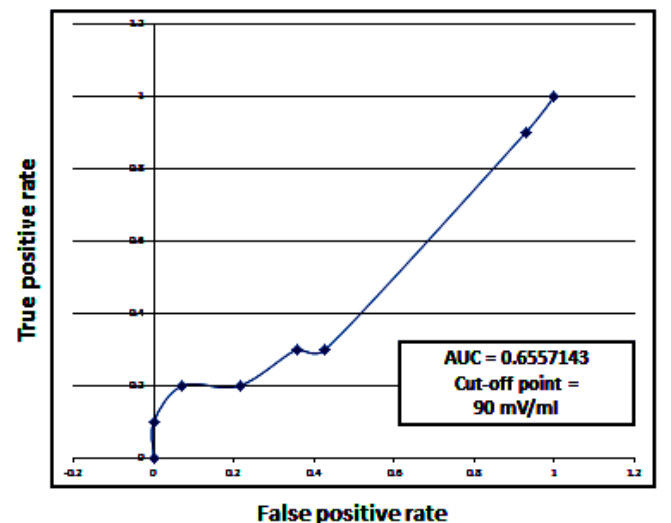

Fig 6: ROC curve for ORP in the follicular fluid as a predictor of pregnancy.

\section{ORP in the spent culture medium}

The ORP in the spent culture medium was studied to evaluate the possibility of its use as a non-invasive method for embryo selection. The mean $( \pm \mathrm{SD})$ ORP in the spent culture medium of oocytes which fertilized $(213.82 \mathrm{mV} / \mathrm{ml} \pm 49.85)$ was lower than in those which did not fertilize $(223.97 \mathrm{mV} / \mathrm{ml} \pm 5.18)$. However, this difference was not statistically significant $(\mathrm{P}=0.4984)$. Similarly, the mean $( \pm \mathrm{SD})$ ORP in the spent culture medium of women who became pregnant $(215.78 \mathrm{mV} / \mathrm{ml} \pm 38.52)$ was lower than in those who did not become pregnant $(229.22$ $\mathrm{mV} / \mathrm{ml} \pm 31.56$ ) but the difference was also not statistically significant $(\mathrm{P}=0.3170)$.

Finally, the ORP in the spent culture medium of individually cultured embryos was evaluated as a predictor of pregnancy. Receiver operating characteristic curve analysis was conducted and the area under the curve was found to be equal to 0.7143 and the cut-off point at $220 \mathrm{mV} / \mathrm{ml}$ (Figure 7).

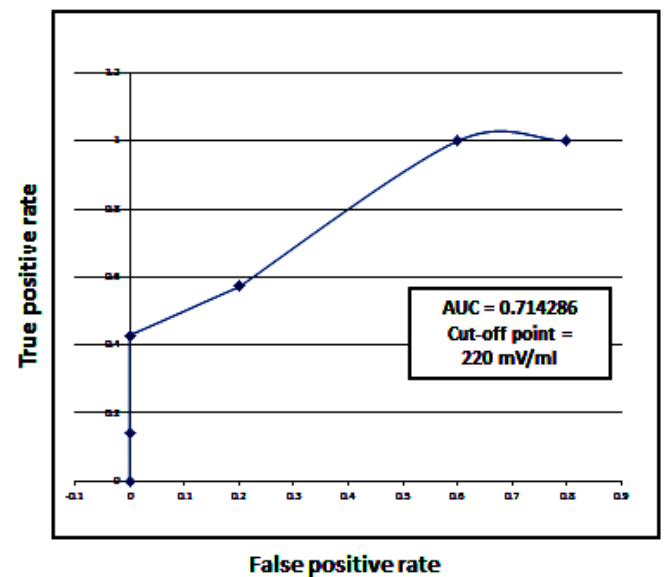

Fig 7: ROC for ORP in the spent culture medium as a predictor of pregnancy.

\section{Predictors of fertilization and pregnancy in IVF}

Finally, the studied parameters were compared regarding the ability to predict $50 \%$ fertilization of the oocytes in IVF by comparing the AUC in the ROC curves. It was found that the ORP in the native semen was the best predictor of fertilization (AUC = $0.854167)$, followed by the in the follicular fluid $(\mathrm{AUC}=0.659259)$.

The studied parameters were also compared regarding the ability to predict pregnancy in IVF by comparing the AUC in the ROC curves. It was found that the embryo score was the best predictor of pregnancy $(\mathrm{AUC}=0.931818)$, followed by the ORP in the native semen $(\mathrm{AUC}=0.8000)$, the ORP in the spent culture medium $(\mathrm{AUC}=0.714286)$.

\section{DISCUSSION}

For many years oxidative stress in semen was blamed as a cause of male infertility. However, this suggestion was based on indirect evidence, such as finding elevated ROS levels in the semen of infertile men ${ }^{3.6,7,8}$ or finding a good correlation between the oxidative stress markers in semen (ROS or TAC) and other markers of male infertility in the semen, namely sperm motility and strict sperm morphology $9,10,11$. Indirect evidence was also provided by the positive relation between high ROS levels in semen on one hand and sperm DNA damage $^{12}$ and apoptosis $^{13}$ on the other hand. However, the effect of oxidative stress on the sperm fertilizing capacity can only be directly evaluated by measuring oxidative stress in the actual native semen sample used for fertilizing the oocytes in the IVF model (not the ICSI model), which offers an ideal set-up for providing a direct answer to this important issue.

The results of the current study have demonstrated that native semen with increased ORP is indeed 
associated with low fertilization rates in IVF and this was confirmed by 2 experiments. Firstly, there was a negative correlation between the ORP in the native semen and the fertilization rate in IVF, and secondly, the ORP in the native semen of patients who achieved $=>50 \%$ fertilization rate was significantly lower than in those who achieved $<50 \%$ fertilization rate. ROC curves were then constructed to evaluate the test as a predictor of $=>50 \%$ fertilization, and the cut-off point was found to be $1.57 \mathrm{mV} / 10^{6} \mathrm{sperm} / \mathrm{ml}$. The current study, therefore, offers direct evidence that oxidative stress in the native semen is detrimental to the fertilizing capacity of the sperm. In addition, ORP in native semen was also found to be significantly lower in the native semen of men who achieved pregnancy by IVF compared to those who did not. The ROC curve showed that the test was a good predictor of pregnancy with a cut-off level of $0.75 \mathrm{mV} / 10^{6} \mathrm{sperm} / \mathrm{ml}$.

These findings support the use of antioxidants in the treatment of infertile men ${ }^{14,15,16,17}$. Based on the current findings, a test for the measurement of oxidative stress in the semen of infertile men is advised before starting antioxidant therapy, if the ORP is found to be $>1.57 \mathrm{mV} / 10^{6} \mathrm{sperm} / \mathrm{ml}$. In addition, this treatment should be monitored by repeating the test at intervals for fear of exceeding the dose of antioxidants leading to reductive stress which is equally detrimental ${ }^{18,19}$. And although the evaluation of oxidative stress in the past needed a labor intensive and costly test, the new platforms for the evaluation of ORP offer simpler, more costeffective and more practical tests ${ }^{20}$.

The effect of oxidative stress in the follicular fluid on the oocytes and resulting embryos and its relation to the fertilization and pregnancy rates were also studied. There was a positive and highly significant correlation between the ORP levels in the follicular fluid pool and the fertilization rate. In addition, the mean ORP levels in the follicular fluid pool of women who achieved $=>50 \%$ fertilization rate was higher than those who did not. On the contrary, there was no significant difference in the follicular fluid pool ORP of women who became pregnant and those who did. This apparent controversy reflects exactly was has been published in the medical literature. For example, Pekel et al measured malondialdehyde (MDA), superoxide dismutase (SOD) and total antioxidant capacity (TAC) in the follicular fluid of infertile patients undergoing IVF and found that antioxidant activity was impaired in these patients compared to controls, possibly implying increased apoptosis ${ }^{21}$. Similar findings had been reported earlier by Oyawoye et al. These workers showed that embryos that survived to transfer came from follicles that had significantly lower total antioxidant capacity (TAC) i.e. under some oxidative stress ${ }^{22}$.

This paradox can also be explained by the fact that, although the pre-ovulatory processes and ovulation are associated with some degree of oxidative stress reflecting increased metabolism, healthy oocytes which eventually achieve pregnancy are those which are better equipped to cope with this oxidative stress (e.g. by having superior anti-oxidant capacity). Other less healthy oocytes unable to cope with the oxidative stress may just be able to achieve fertilization but fail to implant and achieve a pregnancy. The mean ORP in the follicular fluid pool was also evaluated as a predictor of fertilization and pregnancy in IVF. ROC curves were constructed and found that it was a fair predictor of both events.

The results show that the mean ORP in the spent culture medium was also lower in the oocytes which fertilized versus those which did not fertilize. Similarly, the mean ORP was also lower in the culture medium of embryos that achieved pregnancy by IVF versus those which did not achieve pregnancy. These findings are in line with those of Bedaiwy et al who analyzed the culture medium on Day 3 and showed that embryos that resulted in a pregnancy showed significant lower ROS levels than embryos that did not yield a pregnancy ${ }^{23}$. It has therefore been suggested that the supplementation of the culture medium with antioxidants may help reduce this oxidative stress. These include serum albumin as well as glutathione, glutamine or hypotaurine ${ }^{24,25}$. Indeed, the addition of antioxidants to the culture media resulted in an increase in implantation rate and pregnancy rate for patients 3540 years, presumably by reducing oxidative stress ${ }^{26}$.

On the other hand, our findings do not agree with those of Wiener-Megnazi et al (2011) and those of Alegre et al (2019) who found that oxidative stress markers were higher in the embryos which achieved better development compared to those which did $\operatorname{not}^{27,28}$. This controversy can be explained by the fact that these studies used different culture media. Indeed, some culture media are supplemented with serum or serum substitutes, albumin, vitamins, or buffers, which could be a source of $\operatorname{ROS}^{29}$. On the other hand, some culture media are supplemented with antioxidants and the amount of antioxidants added varies from culture medium to another. In fact, PannerSelvamet al found a large discrepancy in the ORP of various culture media and the Sage Step-one medium was found to have the least baseline ORP compared to other commercial brands ${ }^{30}$. Unfortunately, the exact composition of the culture media is an industrial secret and the type and amount of antioxidant added in each culture medium is difficult to determine.

Based on the above mentioned findings, oxidative stress in the culture medium has been suggested as a method for embryo selection in IVF. In 2011, Wiener-Megnazi et al measured ROS in the spent culture medium of embryos by chemiluminescence and suggested that the method was a possible tool for improved embryo selection in IVF $^{27}$ More recently,Alegre et al suggested the combination of the measurement of ROS in the culture medium with time-lapse evaluation of morphokinetic criteria as a method of embryo selection ${ }^{28}$. In both cases, ROS was measured by chemiluminescence. Despite the discrepancy, the findings of the current study confirm this suggestion and show that the measurement of 
ORP in the spent culture medium is an even simpler and less time-consuming method.

Finally, an attempt was made to compare the various predictors of fertilization and pregnancy studied in the work. It was found that the ORP in the native semen was the best predictor of fertilization and this lends support to the theory that ORP in the native semen is an important index of the fertilizing capacity of the sperm and to the suggestion that the measurement of ORP should be included in the evaluation of the infertile male, in any semen analysis ${ }^{4,31,32}$. On the other hand, when predictors of pregnancy were compared, the Veeck embryo score was the best predictor of pregnancy followed by the ORP in the native semen, the ORP in the spent culture medium and finally the ORP in the follicular fluid. This finding is in line with most of the published literature ${ }^{33.34 .35 .36}$.

\section{CONCLUSION}

In conclusion, this study confirms the important role of oxidative stress in assisted reproduction and throws further light on its specific role in each step of the procedure.ORP in the native semen and in the spent culture medium in IVF are good predictors of fertilization and clinical pregnancy while ORP in the follicular fluid is not.

\section{REFERENCES}

1. De GeyterCh, Calhaz-Jorge C, KupkaMS,et al. ART in Europe, 2014: results generated from European registries by ESHRE: The European IVF-monitoring Consortium (EIM) for the European Society of Human Reproduction and Embryology (ESHRE). Hum Reprod. 2018;33(9):1586-1601.

2. SART, National Summary Report, $C D C$, Atlanta, Georgia, 2016. https://www.cdc.gov/art/pdf/2016national-summary- slides/ART_2016_ graphs_ and_charts. pdf

3. Tremellen K. Oxidative stress and male infertility: A clinical perspective. HumReprodUpdate. 2008; 14: $243-58$.

4. Agarwal A and Bui AD. Oxidation-reduction potential as a new marker for oxidative stress: Correlation to male infertility. InvestigClinUrol. 2017;58:385-99.

5. MacLeod J. The role of oxygen in the metabolism and motility of human spermatozoa. Am J Physiol. $1943 ; 138: 512-8$.

6. Iwasaki A and Gagnon C. Formation of reactive oxygen species in spermatozoa of infertile patients. Fertil Steril. 1992; 57:409-16.

7. Pasqualotto FF, Sharma RK, Nelson DR, et al. Relationship between oxidative stress, semen characteristics, and clinical diagnosis in men undergoing infertility investigation. Fertil Steril 2000; 73:459-64.

8. Agarwal A, Gupta S and Sikka S. The role of free radicals and antioxidants in reproduction. Current OpinionObstetGynecol. 2006; 18:325-32.

9. Agarwal A, Saleh RA and Bedaiwy MA. Role of reactive oxygen species in the pathophysiology of human reproduction. Fertil Steril. 2003; 79(4):829-43.

10. Aziz N, Saleh RA, Sharma RK, et al. Novel association between sperm reactive oxygen species production, sperm morphological defects, and sperm deformity index. Fertil Steril. 2004; 81:349-54.

11. Agarwal A, Sharma RK, Sharma R, et al. Characterizing semen parameters and their association with reactive oxygen species in infertile men. ReprodBiolEndocrinol. 2014; 12:33.

12. Desai N, Sharma R, Makker K, et al. Physiologic and pathologic levels of reactive oxygen species in neat semen of infertile men. Fertil Steril. 2009; 92:1626-31.

13. Agarwal A and Said TM. Oxidative stress, DNA damage and apoptosis in male infertility: a clinical approach. BJU Int. 2005; 95:503-7.

14. Comhaire FH, Christophe AB, Zalata AA, et al. The effects of combined conventional treatment, oral antioxidants and essential fatty acids on sperm biology in subfertile men. Prostaglandins LeukotEssent Fatty Acids. 2000; 63:159-65.

15. Gharagozloo P and Aitken RJ. The role of sperm oxidative stress in male infertility and the significance of oral antioxidant therapy. Hum Reprod. 2011; 26:1628-40.

16. Majzoub A and Agarwal A. Systematic review of antioxidant types and doses in male infertility: Benefits on semen parameters, advanced sperm function, assisted reproduction and live-birth rate. Arab J Urol. 2018; 16(1):113-24.

17. Smits RM, Mackenzie-Proctor R, Yazdani A, et al. Antioxidants for male subfertility. Cochrane Database Syst Rev. 2019; 3(3):7411

18. HenkelR, Sandhu IS and Agarwal A. The excessive use of antioxidant therapy: A possible cause of male infertility? Andrologia. 2019; 51(1):13162.

19. PannerSelvam MK, Henkel R, Sharma R, et al. Calibration of redox potential in sperm wash media and evaluation of oxidation-reduction potential values in various assisted reproductive technology culture media using MiOXSYS system. Andrology. 2018; 6(2):293-300.

20. Agarwal A, Sharma R, Shubhadeep Roychoudhury S, et al. MiOXSYS: a novel method of measuring oxidation reduction potential in semen and seminal plasma. Fertil Steril. 2016;106(3):566-73.

21. Pekel A, Gönenç A, Turhan NO, et al. Changes of sFas and sFasL, oxidative stress markers in serum and follicular fluid of patients undergoing IVF. $J$ Assist Reprod Genet. 2015; 32(2):233-41.

22. Oyawoye O A, Abdel Gadir A, Garner N, et al. Antioxidants and reactive oxygen species in follicular fluid of women undergoing IVF: relationship to outcome. Hum Reprod. 2003; 18(11): 2270-4.

23. Bedaiwy MA, Mahfouz RZ, Goldberg JM, et al. Relationship of reactive oxygen species levels in day 3 culture media to the outcome of in vitro fertilization/intracytoplasmic sperm injection cycles. Fertil Steril. 2010; 94(6):2037-42.

24. Ozawa M, Nagai T, Fahrudin M, et al. Addition of glutathione or thioredoxin to culture medium reduces intracellular redox status of porcine IVM/IVF embryos, resulting in improved 
development to the blastocyst stage. MolReprodDev. 2006;73(8):998-1007.

25. Suzuki C, Yoshioka K, Sakatani M, et al. Glutamine and hypotaurine improves intracellular oxidative status and in vitro development of porcine preimplantation embryos. Zygote. 2007;15(4): 317-24.

26. Gardner DK, Kuramoto T, Tanaka M, et al. Prospective randomized multicentre comparison on sibling oocytes comparing G-Series media system with antioxidants versus standard G-Series media system. Reprod Biomed Online. 2020; 40(5):637-44.

27. Wiener-Megnazi Z, Shiloh H, Avraham L, et al. Oxidative parameters of embryo culture media may predict treatment outcome in in vitro fertilization: a novel applicable tool for improving embryo selection. Fertil Steril. 2011; 95(3):97984.

28. Alegre L, Del Gallego R, Arrones S, et al. Novel noninvasive embryo selection algorithm combining time-lapse morphokinetics and oxidative status of the spent embryo culture medium. Fertil Steril 2019;111(5):918-27.

29. Martin-Romero FJ, Miguel-Lasobras EM, Dominguez-Arroyo JA, et al. Contribution of culture media to oxidative stress and its effect on human oocytes. Reprod Biomed Online. 2008, 17:652-61

30. PannerSelvam MK, Agarwal A, Henkel R, et al. The effect of oxidative and reductive stress on semen parameters and functions of physiologically normal human spermatozoa. Free RadicBiol Med $2020 ; 152: 375-85$

31. Agarwal A and Wang SM. Clinical relevance of oxidation-reduction potential in the evaluation of male infertility. Urology. 2017; 104:84-9.

32. Agarwal A, Roychoudhury S, Sharma R, et al. Diagnostic application of oxidation-reduction potential assay for measurement of oxidative stress: clinical utility in male factor infertility. Reprod Biomed Online. 2017; 34(1):48-57.

33. Ebner T, Moser M, Sommergruber M, et al. Selection based on morphological assessment of oocytes and embryos at different stages of preimplantation development: a review. Hum Reprod Update. 2003; 9(3):251-62

34. Rhenman A, Berglund L, Brodin T, et al. Which set of embryo variables is most predictive for live birth? A prospective study in 6252 single embryo transfers to construct an embryo score for the ranking and selection of embryos. Hum Reprod. 2015; 30(1):28-36.

35. Simopoulou M, Sfakianoudis K, Antoniou N, et al. Making IVF more effective through the evolution of prediction models: is prognosis the missing piece of the puzzle?. SystBiolReprod Med. 2018; 64(5):305-23

36. vanLoendersloot $\mathrm{L}$, van Wely $\mathrm{M}$, van der Veen $\mathrm{F}$, et al. Selection of embryos for transfer in IVF: ranking embryos based on their implantation potential using morphological scoring. Reprod Biomed Online. 2014; 29(2):222-30. 